\title{
On Controlling Light Transport in Poor Visibility Environments
}

\author{
Mohit Gupta, Srinivasa G. Narasimhan \\ Carnegie Mellon University \\ School of Computer Sc., Pittsburgh 15232, USA \\ \{mohitg, srinivas\}ecs.cmu.edu
}

\author{
Yoav Y. Schechner \\ Technion - Israel Institute of Technology \\ Dep. of Electrical Eng., Haifa 32000, Israel \\ yoaveee.technion.ac.il
}

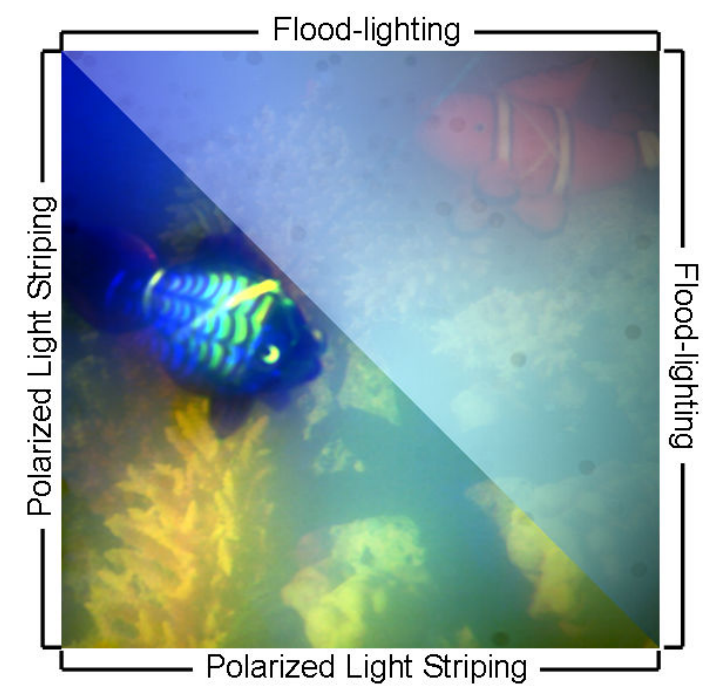

Figure 1. Polarized light striping versus flood-lighting. In this experiment, the scene is comprised of objects immersed in murky water. Using the polarized light striping approach, we can control the light transport before image formation for capturing the same scene with better color and contrast. High-resolution images can be downloaded from the project web-page [7].

scene comprised of objects immersed in murky water.

While propagating within a medium such as murky water or fog, light gets absorbed and scattered. Broadly speaking, light transport [2] can be classified based on three specific pathways: (a) from the light source to the object, (b) from the object to the sensor and (c) from the light source to the sensor without reaching the object (see Figure 2). Of these, the third pathway causes loss of contrast and effective dynamic range (for example, the backscatter of car headlights in fog), and is thus undesirable.

We wish to build active illumination and sensing systems that maximize light transport along the first two pathways while simultaneously minimizing transport along the third. To this end, we exploit some real world observations. For example, while driving in foggy conditions, flood-lighting the road ahead with a high-beam may reduce visibility due to backscatter. On the other hand, underwater divers realize that maintaining a good separation between the source and the camera reduces backscatter, and improves visibil- 


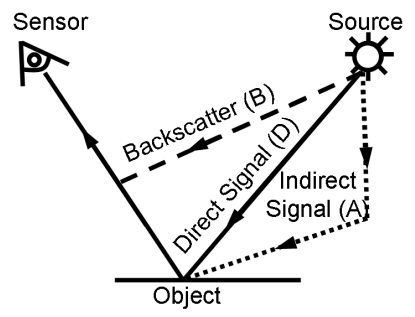

(a)

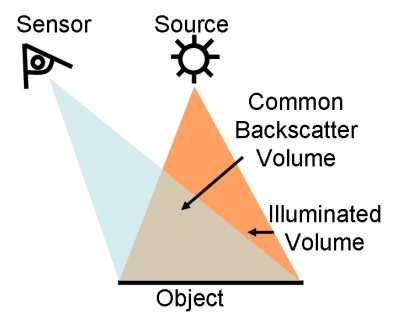

(b)

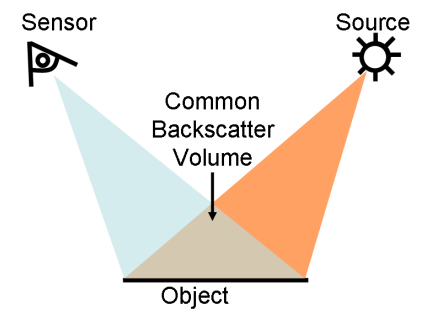

(c)

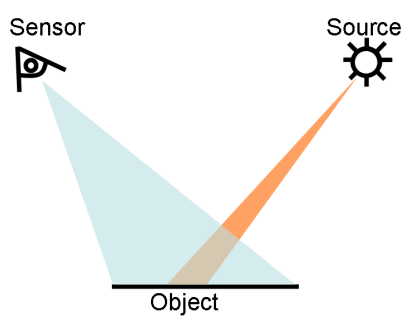

(d)

Figure 2. Light transport in scattering media for different source and sensor configurations. (a) Illustration of the three light transport components. (b) The backscatter $B$ reduces the image contrast. The amount of backscatter increases with the common backscatter volume. (c) By changing the relative placement of the sensor and source, we can modulate the light transport components for increasing the image contrast. (d) The common backscatter volume can be reduced by using light stripe scanning as well.

ity [21, 9]. Polarization filters have also been used to reduce contrast loss due to haze and murky water [20, 24, 19, 6]. Based on these observations, we attempt to address two key questions. First, which illumination and sensing modality allows us to modulate the three light transport pathways most effectively? Second, what is the "optimal" placement of the source and the sensor? This paper has two main contributions:

(1) We present an active imaging technique called polarized light striping and show that it performs better than previous techniques such as flood-lighting, unpolarized light striping [10, 15, 9], and high frequency illumination based separation of light transport components [16].

(2) We derive a numerical approach for computing the optimal relative sensor-source position in poor visibility conditions. We consider a variety of illumination and sensing techniques, while accounting for the limits imposed by sensor noise. Our model can be used for improving visibility in different outdoor applications. It is useful for tasks such as designing headlights for vehicles (terrestrial and underwater). We validate our approach in real experiments.

\section{How to Illuminate and Capture the Scene?}

In this section, we present an active imaging technique: polarized light striping. We also analyze the relative merits of different existing techniques, and show that polarized light striping outperforms them.

While propagating through a medium, light gets absorbed and scattered (Figure 2). The image irradiance at a particular pixel is given as a sum of the three components, the direct signal $(D)$, the indirect signal $(A)$ and the backscatter $(B)$ :

$$
E(x, y)=\underbrace{D(x, y)+A(x, y)}_{\text {Signal }}+\underbrace{B(x, y)}_{\text {Backscatter }} .
$$

The total signal $S$ is

$$
S(x, y)=D(x, y)+A(x, y) .
$$
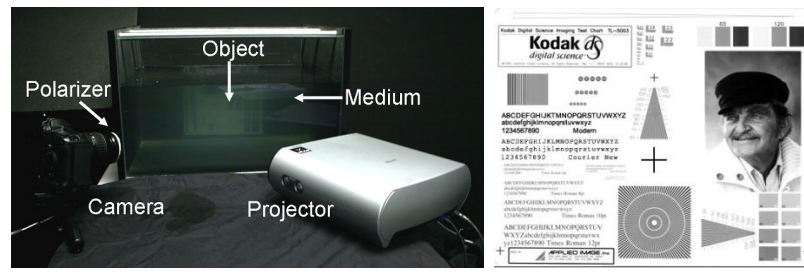

Experimental Setup
Kodak Contrast Chart
Figure 3. Our experimental setup consisting of a glass tank, filled with moderate to high concentrations of milk (four times as those in [15]). An LCD projector illuminates the medium with polarized light. The camera (with a polarizer attached) observes a contrast chart through the medium.

The backscatter $B$ degrades visibility and depends on the optical properties of the medium such as the extinction coefficient and the phase function. The direct and the indirect components ( $D$ and $A$ ) depend on both the object reflectance and the medium. Our goal is to design an active illumination and sensing system that modulates the components of light transport effectively. Specifically, we want to maximize the signal $S$, while minimizing the backscatter $B$.

We demonstrate the effectiveness of different imaging techniques in laboratory experiments. Our experimental setup consists of a $60 \times 60 \times 38 \mathrm{~cm}^{3}$ glass tank filled with dilute milk (see Figure 3). The glass facades are antireflection coated to avoid stray reflections. ${ }^{1}$ The scene consists of objects immersed in murky water or placed behind the glass tank. A projector illuminates the scene and a camera fitted with a polarizer observes the scene. We use a Sony VPL-HS51A, Cineza 3-LCD video projector. The red and the green light emitted from the projector are inherently polarized channels. If we want to illuminate the scene with blue light, we place a polarizer in front of the projector. We use a 12-bit Canon EOS1D Mark-II camera, and a Kodak contrast chart as the object of interest to demonstrate the contrast loss or enhancement for different techniques.

\footnotetext{
${ }^{1}$ Imaging into a medium through a flat interface creates a non-single viewpoint system. The associated distortions are analyzed in [25].
} 


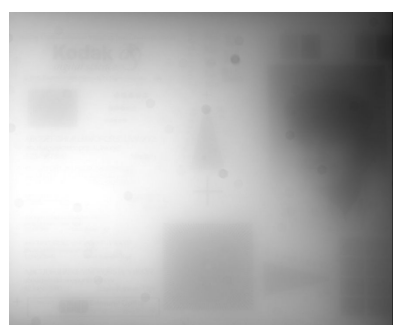

(a) Maximum image

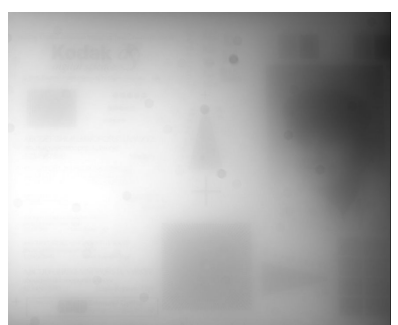

(b) Global component

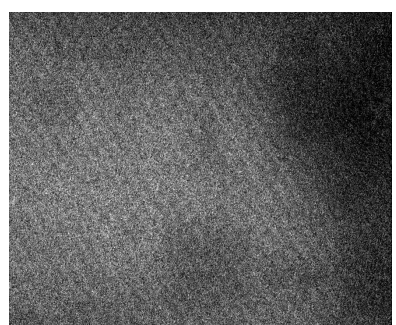

(c) Direct component

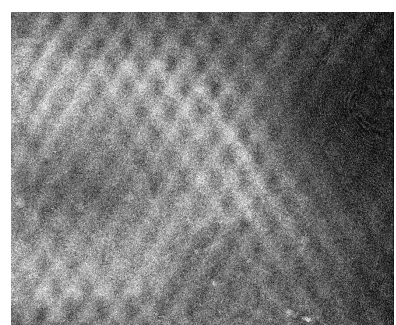

(d) Direct component (low freq)

Figure 4. Limitations of the high frequency illumination based method. A shifting checkerboard illumination pattern was used with the checker size of $10 \times 10$ pixels. (a) Maximum image (b) Minimum image (global component) (c) Direct component (d) Direct component obtained using lower frequency illumination (checker size of $20 \times 20$ pixels). The direct component images have low SNR in the presence of moderate to heavy volumetric scattering. The global image is approximately the same as a flood-lit image, and hence, suffers from low contrast. This experiment was conducted in moderate scattering conditions, same as the second row of Figure 6.

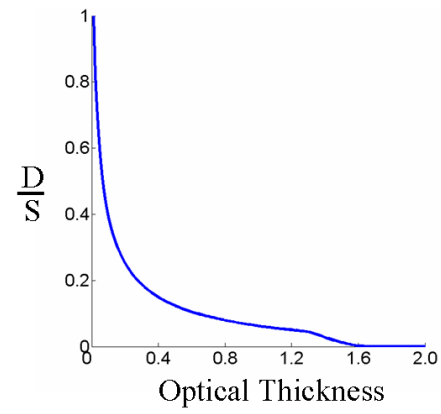

Figure 5. The relative direct component of the signal reduces with increasing optical thickness of the medium. This plot was calculated using simulations, with a two-term Henyey-Greenstein scattering phase function [8] for a parameter value of 0.8 .

High-frequency illumination: Ref. [16] presented a technique to separate direct and global components of light transport using high frequency illumination, with good separation results for inter-reflections and sub-surface scattering. What happens in the case of light transport in volumetric media? Separation results in the presence of moderate volumetric scattering are illustrated in Figure 4. The direct component is the direct signal $(D)$, whereas the global component is the sum of indirect signal $(A)$ and the backscatter $(B)$, as shown in Figure 2. Thus, this method seeks the following separation:

$$
E(x, y)=\underbrace{D(x, y)}_{\text {Direct }}+\underbrace{A(x, y)+B(x, y)}_{\text {Global }} .
$$

However, to achieve the best contrast, we wish to separate the signal $D+A$ from the backscatter $B$. As the medium becomes more strongly scattering, the ratio $\frac{D}{S}$ falls rapidly due to heavy attenuation and scattering, as illustrated in Figure 5. This plot was estimated using numerical simulations using the single scattering model of light transport. ${ }^{2}$ Consequently, for moderate to high densities of the

\footnotetext{
${ }^{2}$ With multiple scattering, the ratio falls even more sharply.
}

medium, the direct image suffers from low signal-to-noiseratio (SNR), as shown in Figure 4. Further, the indirect signal $(A)$ remains unseparated from the backscatter $B$, in the global component. Thus, the global image is similar to a flood-lit image, and suffers from low contrast.

Polarized flood-lighting: Polarization imaging has been used to improve image contrast $[19,23,6]$ in poor visibility environments. It is based on the principle that the backscatter component is partially polarized, whereas the scene radiance is assumed to be unpolarized. Using a sensor mounted with a polarizer, two images can be taken with two orthogonal orientations of the polarizer:

$$
\begin{aligned}
E_{\mathrm{b}} & =\frac{D+A}{2}+\frac{B(1-p)}{2} \\
E_{\mathrm{w}} & =\frac{D+A}{2}+\frac{B(1+p)}{2},
\end{aligned}
$$

where $p$ is the degree of polarization (DOP) of the backscatter. Here, $E_{\mathrm{b}}$ and $E_{\mathrm{w}}$ are the 'best-polarized image' and the 'worst-polarized image', respectively. Thus, using optical filtering alone, backscatter can be removed partially, depending on the value of $p$. Further, it is possible to recover an estimate of the signal $\hat{S}$ in a post-processing step [19]:

$$
\hat{S}=E_{\mathrm{b}}\left(1+\frac{1}{p}\right)+E_{\mathrm{w}}\left(1-\frac{1}{p}\right) .
$$

However, in optically dense media, heavy backscatter due to flood-lighting can dominate the signal, making it impossible for the signal to be recovered. This is illustrated in Figure 6, where in the case of flood-lighting under heavy scattering, polarization imaging does not improve visibility.

Light stripe scanning: Here, a thin sheet of light is scanned across the scene. In comparison to the above approaches, the common backscatter volume is considerably 


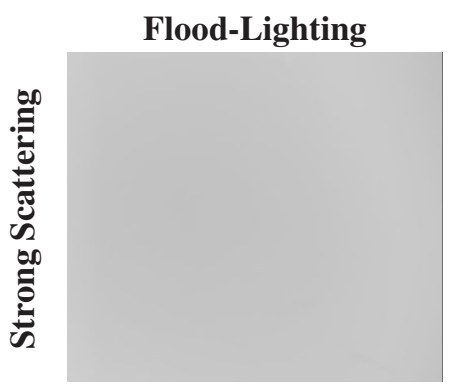

(a)

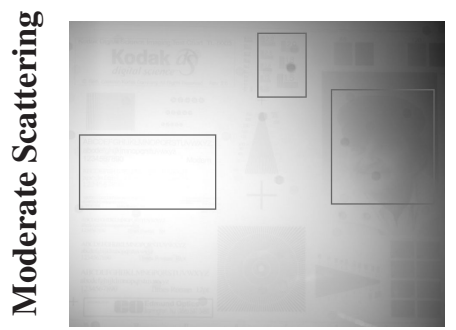

(e)

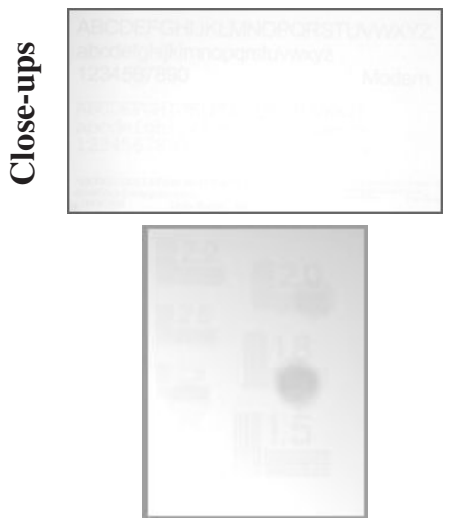

(i)

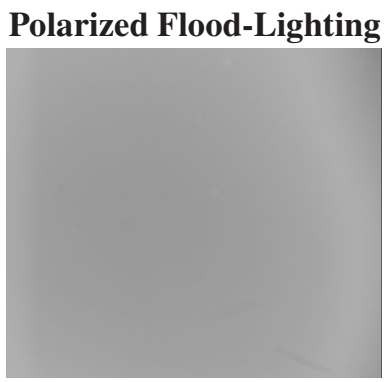

(b)

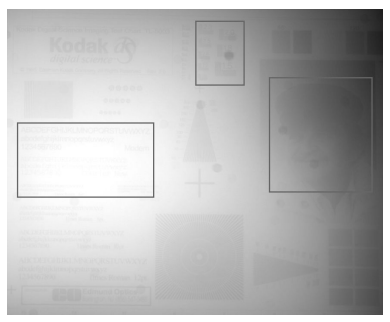

(f)

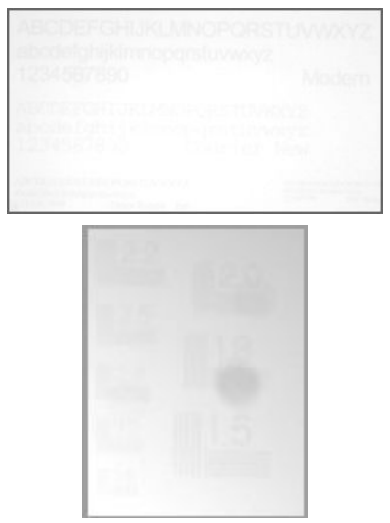

(j)

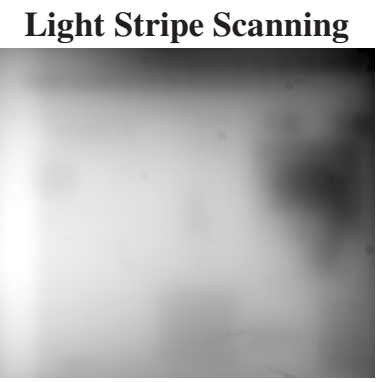

(c)

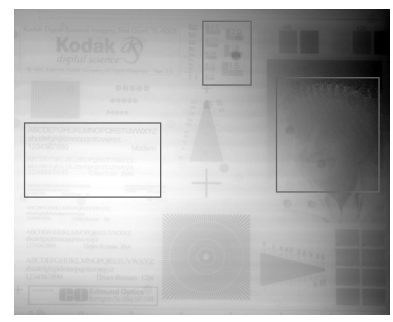

(g)

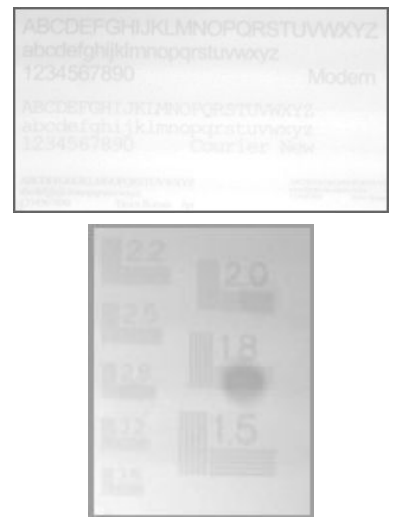

(k)

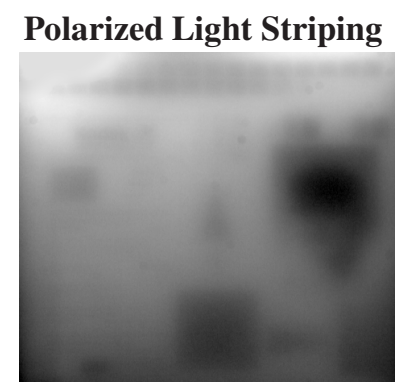

(d)

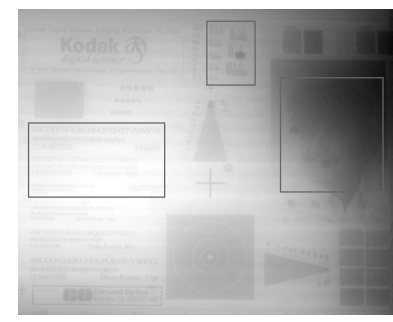

(h)

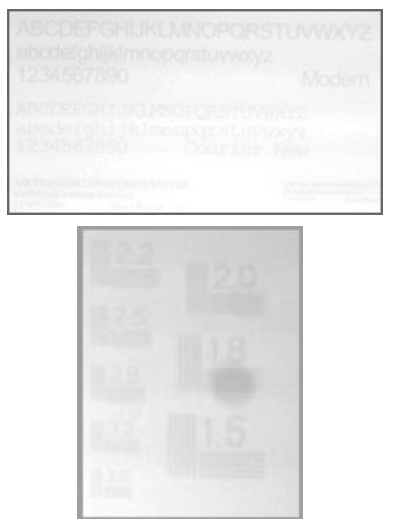

(1)

Figure 6. Comparison of various illumination and sensing techniques (zoom into the marked areas to better assess the image quality). Flood-lit images suffer from a severe loss of contrast, specially in the presence of heavy scattering (a,b). Polarized light striping achieves a significant increase in image contrast, even in the presence of heavy scattering (a-d). In moderate scattering, fine details (text) are recovered more reliably in (g) and (h), as compared to (e). See (i), (j), (k) and (l) for close-ups of the marked areas in (e), (f), (g) and (h) respectively. The moderate scattering experiment was conducted under the same conditions as the experiment in Figure 4.

reduced (see Figure 2d). The sheet of light intersects the object to create a stripe that is detected using a gradient operator. ${ }^{3}$ All stripes are then mosaiced to create a composite image CI [10, 15, 9]. Alternatively, the composite image can be obtained by simply selecting the maximum value at each pixel over all the individual light stripe images $\mathrm{SI}_{k}$ :

$$
\mathrm{CI}(x, y)=\max _{k}\left\{\operatorname{SI}_{k}(x, y)\right\}
$$

\footnotetext{
${ }^{3}$ In our particular implementation, the projector illuminates a single plane and has low power. We compensate for this by increasing the exposure time of the camera.
}

Polarized light striping: We propose polarized light striping as a technique that combines the advantages of polarization imaging and light striping, and thus, is applicable for an extended range of medium densities. Earlier, we demonstrated that light striping reduces the amount of backscatter. However, reliable localization of the object stripes (by using gradient operator or by selecting the maximum pixel value, as in Eq. 7) is severely impeded due to strong backscatter. This is illustrated in Figure 7.

To enable reliable detection of the object stripes even in the presence of strong scattering, we use polarization imaging in conjunction with light striping. A high DOP of the 


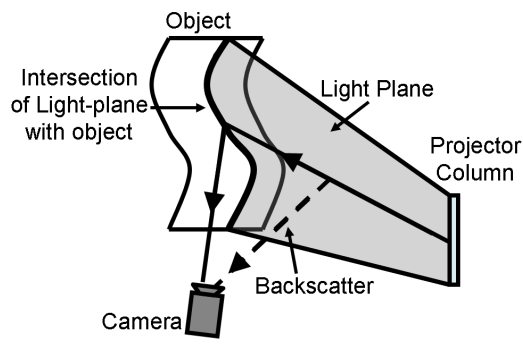

(a)

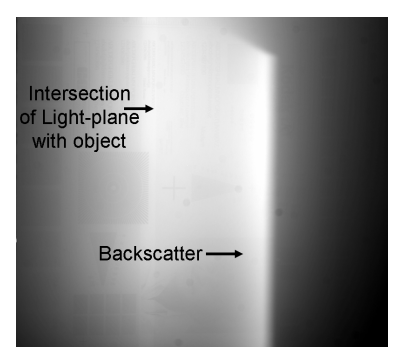

(b)

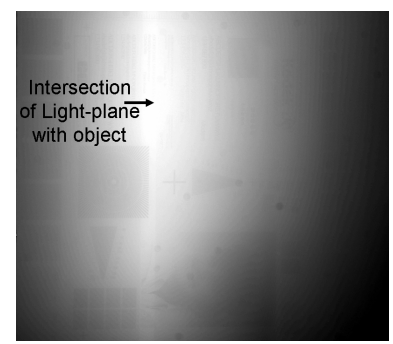

(c)

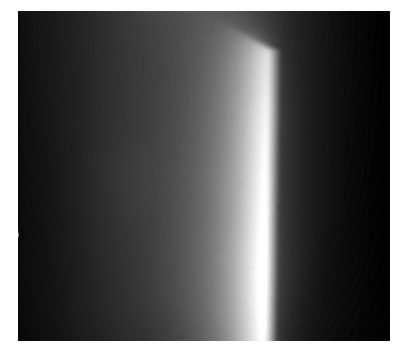

(d)

Figure 7. Unpolarized versus polarized light stripe scanning. (a) Ray diagram illustrating light stripe scanning, adapted from [15]. (b) The camera observes a light stripe ( 1 out of 30 ) without a polarizer. The visible light plane is the backscatter and impedes reliable detection of the object stripe. (c) Through a polarizer, there is a considerable reduction in backscatter. The light plane-object intersection becomes more distinct, thus enabling its reliable delineation. (d) The removed backscatter (difference of (b) and (c)). Video of a complete scan can be downloaded from the project web-page [7].

backscatter is essential for removing the backscatter using polarization filtering (Eq. 4), or to recover a reliable estimate of the signal using post-processing (Eq. 6). In our experiments, the camera observes the scene through a polarization filter and the light sheets irradiating the scene are polarized. Since the incident illumination is completely polarized, the DOP of the backscatter is high (see appendix). This results in a significant reduction in the amount of backscatter, and thus, enables reliable detection of the stripes. ${ }^{4}$ This is shown in Figure 7. We compare the results of polarized light striping versus previous illumination and sensing techniques in Figure 6. Notice especially the differences in the contrast under strong scattering. Another result is shown in Figure 1.

\section{Optimal Camera-Source Placement}

Conventional wisdom from the underwater imaging literature suggests maximizing the sensor-source separation to reduce the backscatter, and hence, increase the image contrast $[9,21]$ (see Figure 2). However, this does not take into account the limitations posed by measurement noise. Indeed, placing the source and the sensor far from each other or the scene results in strong attenuation of light, and a low SNR. In this section, we investigate this trade-off between image contrast and SNR to compute the optimal relative sensor-source positions.

\subsection{Quality Measures}

In order to formalize the notion of "optimal", we define various image quality measures for different imaging and illumination techniques. These quality measures serve as objective functions which can be maximized to find the optimal placement of the source and the camera.

Contrast Quality Measure: A major goal of an imaging system is to maximize the image contrast. Analogous to [1,

\footnotetext{
${ }^{4}$ Polarization imaging was previously used with phase-shifted structured illumination for improved reconstruction of translucent objects [3].
}

5], we define the contrast quality measure, $\mathrm{CQM}(x, y)$ as the ratio of the signal $S(x, y)$ to the total intensity $E(x, y)$ :

$$
\operatorname{CQM}(x, y, p)=\frac{S}{S+B(1-p)} .
$$

This measure takes polarization imaging into account by defining the total intensity as that of the best polarized image, as in Eq. (4). In the absence of a polarizer, $p=0$.

Delineation of light plane-scene intersection: Success of light striping in scattering media relies on reliable delineation of the object stripe. One scheme is to detect a brightness discontinuity in the intensity profile across the stripe edge. Thus, for a light stripe scanning system, we define a gradient quality measure (GQM) along the edge of the stripe in terms of the strength of gradient across the stripe edge. Consider Figure 8c; since the scene point $O^{\prime}$ does not have the direct component $D$ or the backscatter component $B(1-p)$, the normalized difference in intensity of $O$ and $O^{\prime}$ is given as:

$$
\operatorname{GQM}(x, y, p)=\frac{D+B(1-p)}{D+A+B(1-p)} .
$$

SNR dependent weighting: An image with high contrast but low overall intensity may result in a low SNR, and hence be of limited use. Thus, we define an SNR dependent weight $\mathrm{W}$ as a monotonically increasing function of the total signal value $S$. The quality measures (CQM and GQM) are weighted by $\mathrm{W}$ so that signal values in the low SNR range are penalized. For example, $\mathrm{W}$ can be a linear function of $S$. For more flexibilty, we use a sigmoid function of $S$ :

$$
\mathrm{W}(x, y)=\frac{1}{1+e^{-\left(\frac{S-\mu}{z}\right)}},
$$

where $\mu$ is the shift and $z$ is the steepness of the sigmoid. For example, $\mu$ can be the dark current offset. Similarly, if the noise is derived from a Gaussian distribution, $z$ can be the standard deviation. In addition, we should account for the effect of post-processing on image noise [24, 17]. 


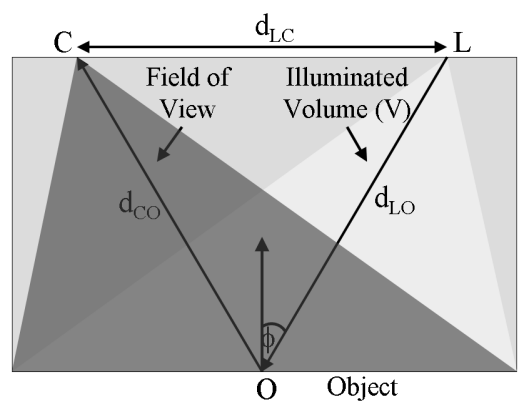

(a)

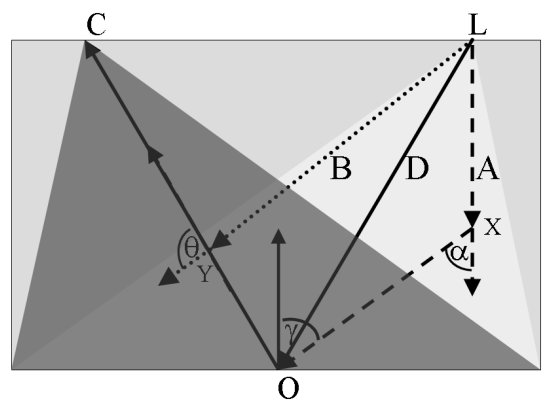

(b)

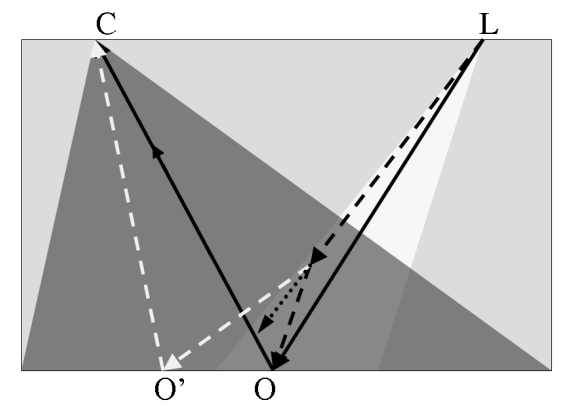

(c)

Figure 8. Simulating image formation for finding the optimal sensor-source configuration. (a) A schematic view of the volume. We use a point light source (L) and a pinhole camera (C). The object is Lambertian, with reflectance $R$. (b) We calculate $D, A$ and $B$ according to Eqs. (11-13). (c) In the case of light striping, the point $O^{\prime}$ is not getting directly irradiated by the source. Also, the viewing ray from $O^{\prime}$ does not intersect the common backscatter volume. Thus, the direct component and the backscatter component at $O^{\prime}$ are null. This results in a brightness gradient across the stripe edge. The strength of the gradient is given by Eq. 9.

\subsection{Simulations}

Consider an underwater scenario where a remote operated vehicle (ROV) wants to capture images at a given distance. Given an approximate estimate of the object albedo, medium scattering parameters [13] and sensor noise, we can simulate the image formation process. To illustrate the concept, we simulate the image formation process for our experimental setup. The Lambertian object reflectance was assumed to be 0.6. For different source-camera configurations, we compute the appropriate quality measure described above. Then, the optimal configuration is the one that maximizes the quality measure.

Figure 8 illustrates the image formation geometry. In our experiments and simulations, the scene and camera remain fixed, while the source is moved to vary the sensor-source separation $d_{\mathrm{LC}}$. Point $O$ on the object is being observed by the camera. Points $X$ and $Y$ are in the medium. The distances $d_{\mathrm{LO}}, d_{\mathrm{CO}}, d_{\mathrm{LX}}, d_{\mathrm{XO}}, d_{\mathrm{CO}}, d_{\mathrm{LY}}$ and $d_{\mathrm{YC}}$, and the angles $\phi, \alpha, \gamma, \theta$ are as illustrated in Figure 8. To keep our simulations simple, we assume a single scattering model of light transport and a homogeneous medium. The individual components of light transport are then given by:

$$
\begin{aligned}
D & =\frac{I_{0}}{d_{\mathrm{LO}}^{2}} e^{-\sigma\left(d_{\mathrm{LO}}+d_{\mathrm{CO}}\right)} R(\phi) \\
A & =\int_{V} \frac{I_{0}}{d_{\mathrm{LX}}^{2}} e^{-\sigma\left(d_{\mathrm{LX}}+d_{\mathrm{XO}}+d_{\mathrm{CO}}\right)} F(\alpha) R(\gamma) d V \\
B & =\int_{O}^{C} \frac{I_{0}}{d_{\mathrm{LY}}^{2}} e^{-\sigma\left(d_{\mathrm{LY}}+d_{\mathrm{YC}}\right)} F(\theta) d Y,
\end{aligned}
$$

where $I_{0}$ is the source radiance, $\sigma$ is the extinction coefficient, $R$ is the Lambertian object reflectance, $F$ is the scattering phase function (we use the two-term HenyeyGreenstein function [8]) and $V$ is the illuminated volume.

Polarized images, $E_{\mathrm{b}}$ and $E_{\mathrm{w}}$ are simulated according to Eqs. (4-5). This requires knowledge of the DOP of the backscatter $p$. Using our experimental setup, we estimated $p$ to be approximately 0.8 , from the regions of the image without any object. We can also compute $p$ analytically, given the dependence of the DOP of scattered light on the scattering angle, such as given in the Appendix.

Optimal configuration for flood-lighting: Let us find the configuration that is optimal in terms of both image contrast and noise. We plot the product of the CQM and $\mathrm{W}$ versus the sensor-source separation $d_{\mathrm{LC}}$ (Figure 9a). The tradeoff between contrast and SNR results in a local maximum. Notice that polarization improves image quality as compared to unpolarized imaging. However, since the DOP (and hence, the amount of contrast enhancement) is similar for all sensor-source positions, the location of the peak remains the same. The curve for the ideal case of zero noise increases monotonically. However, for real world scenarios, where measurement noise places limits on the sensor's abilities, our approach can yield an optimal placement. This is illustrated in Figure 9 (b-c). The image taken using the optimal separation $(40 \mathrm{cms})$ has high contrast and low noise. On the other hand, notice the significant noise in the image taken using a large separation (60 cms).

Optimizing the light stripe scan: The case of light stripe scanning is more interesting. Instead of illuminating the whole scene at once, we illuminate it using one sheet of light at a time. We want to find the optimal light stripe scan. Should we scan the scene (a) by rotating the source, (b) by translating it, or (c) a combination thereof? To answer this, we plot the product of the GQM and the $\mathrm{W}$ for our setup (Figure 10). We observe different optimal separations for different (3 out of 30) stripe locations. Figure 10 (e) shows the high-contrast image acquired using the results of the simulations. The camera and the projector were placed 


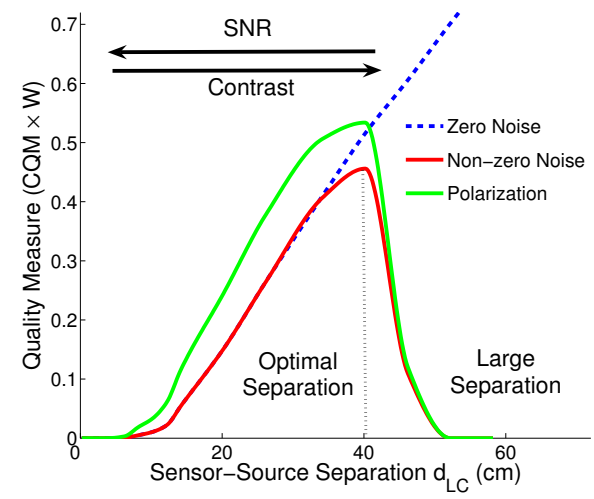

(a)

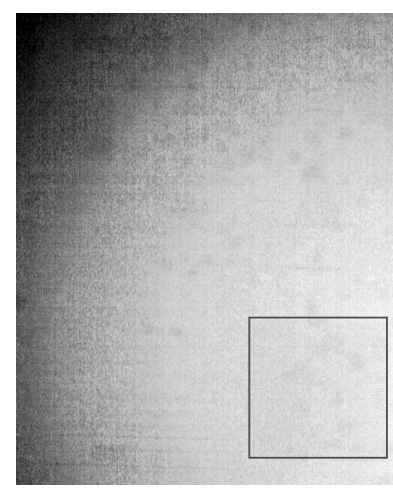

(b) Large Separation

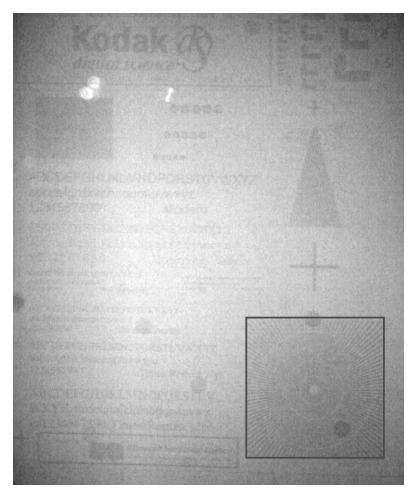

(c) Optimal Separation
Figure 9. Optimal sensor-source configuration for flood-lighting. (a) Plot of $\mathrm{CQM} \times \mathrm{W}$ versus $d_{\mathrm{LC}}$ for our experimental setup. The tradeoff between contrast and SNR results in a maximum. (b) Large separation $(60 \mathrm{cms})$ results in heavy image noise (c) Optimal separation $(40 \mathrm{cms})$ results in a high contrast, low noise image (zoom into the marked area). Both the frames were captured with the same exposure time.

at a small distance from the facade of the glass tank in real experiments. By carefully choosing the light rays, we can simulate a light source and a sensor placed on the glass facade, as assumed in the simulations. The optimal scan for polarized light striping is the same as unpolarized light striping, but results in better image quality.

\section{Discussion}

We study new ways to control light transport for the purpose of capturing better quality data in poor visibility environments. With existing techniques for measurement of medium scattering [13] and polarization properties [27], our simulation-based approach can be used to adapt the illumination and sensing system in-situ. Post-processing approaches are expected to recover the scene when applied to the images acquired using our system. Our analysis focused

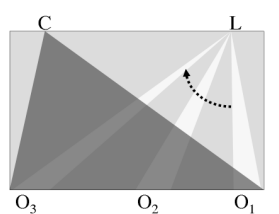

(a)

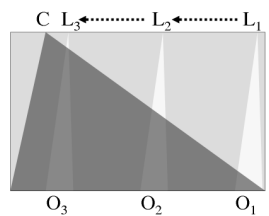

(b)

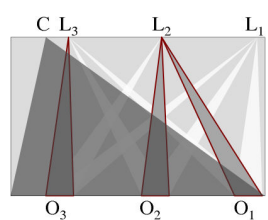

(c)

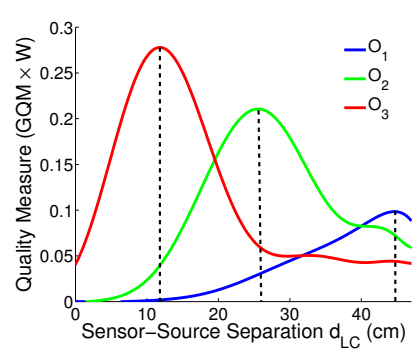

(d)

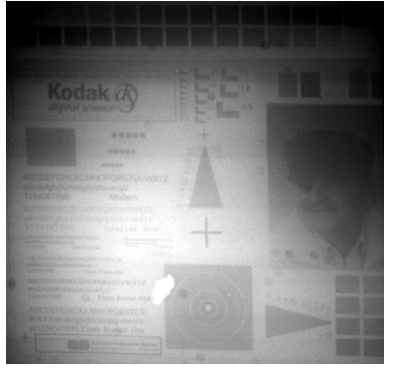

(e)
Figure 10. We can scan the scene (a) by rotating the source, (b) by translating it, or (c) a combination thereof. (d) Plot of GQM $\times$ W versus $d_{\mathrm{LC}}$ for different stripe locations $\mathrm{O}_{1}, \mathrm{O}_{2}$ and $\mathrm{O}_{3}$, for our setup. We can notice different optimal separations for these stripe locations. (e) A high contrast image resulting from the optimal light stripe scan designed using simulations.

on a single divergent source and a single camera. It is worth extending our analysis to multiple cameras and sources [11]. More broadly, we believe that better control of the light transport can be achieved with greater flexibility in choosing illumination and viewing rays.

\section{Acknowledgments}

The authors thank Shahriar Negahdaripour for helpful discussions. This research was supported in parts by Grants \# ONR N00014-08-1-0330, NSF CAREER IIS-0643628, NSF CCF-0541307 and the US-Israel Binational Science Foundation (BSF) Grant \# 2006384. Yoav Schechner is a Landau Fellow - supported by the Taub Foundation. Yoav's work was conducted in the Ollendorff Minerva Center. Minerva is funded through the BMBF.

\section{References}

[1] F. M. Caimi, F. R. Dalgleish, T. E. Giddings, J. J. Shirron, C. Mazel, and K. Chiang. Pulse versus CW laser line scan imaging detection methods: Simulation results. In Proc. IEEE OCEANS, pages 1-4, 2007. 5

[2] S. Chandrasekhar. Radiative Transfer. Dover Publications, Inc., 1960. 1

[3] T. Chen, H. P. A. Lensch, C. Fuchs, and H.-P. Seidel. Polarization and phase-shifting for 3D scanning of translucent objects. In Proc. IEEE CVPR, pages 1-8, 2007. 5

[4] F. Cozman and E. Krotkov. Depth from scattering. In Proc. IEEE CVPR, pages 801-806, 1997. 1 
[5] T. E. Giddings, J. J. Shirron, and A. Tirat-Gefen. EODES-3: An electro-optic imaging and performance prediction model. In Proc. IEEE OCEANS, 2:1380-1387, 2005. 5

[6] G. D. Gilbert and J. C. Pernicka. Improvement of underwater visibility by reduction of backscatter with a circular polarization technique. Applied Optics, 6(4):741-746, 1967. 2, 3

[7] M. Gupta and S. G. Narasimhan. Light transport web-page. http://graphics.cs.cmu.edu/projects/LightTransport/. 1, 5

[8] V. I. Haltrin. One-parameter two-term henyey-greenstein phase function for light scattering in seawater. Applied $\mathrm{Op}$ tics, 41(6):1022-1028, 2002. 3, 6

[9] J. Jaffe. Computer modeling and the design of optimal underwater imaging systems. IEEE Journal of Oceanic Engineering, 15(2):101-111, 1990. 2, 4, 5

[10] D. M. Kocak and F. M. Caimi. The current art of underwater imaging with a glimpse of the past. MTS Journal, 39:5-26, 2005. 2, 4

[11] M. Levoy, B. Chen, V. Vaish, M. Horowitz, I. McDowall, and M. Bolas. Synthetic aperture confocal imaging. ACM Trans. Graph., 23(3):825-834, 2004. 7

[12] S. G. Narasimhan. Models and algorithms for vision through the atmosphere. In Columbia Univ. Dissertation, 2004. 1

[13] S. G. Narasimhan, M. Gupta, C. Donner, R. Ramamoorthi, S. K. Nayar, and H. W. Jensen. Acquiring scattering properties of participating media by dilution. ACM Trans. Graph., 25(3):1003-1012, 2006. 6, 7

[14] S. G. Narasimhan and S. K. Nayar. Contrast restoration of weather degraded images. 25(6):713-724, 2003. 1

[15] S. G. Narasimhan, S. K. Nayar, B. Sun, and S. J. Koppal. Structured light in scattering media. In In Proc. IEEE ICCV, pages 420-427, 2005. 2, 4, 5

[16] S. K. Nayar, G. Krishnan, M. D. Grossberg, and R. Raskar. Fast separation of direct and global components of a scene using high frequency illumination. ACM Trans. Graph., 25(3):935-944, 2006. 2, 3

[17] Y. Y. Schechner and Y. Averbuch. Regularized image recovery in scattering media. IEEE Trans. PAMI, 29(9):16551660, 2007. 5

[18] Y. Y. Schechner and N. Karpel. Recovery of underwater visibility and structure by polarization analysis. IEEE Journal of Oceanic Engineering, 30(3):570-587, 2005. 1

[19] Y. Y. Schechner, S. G. Narasimhan, and S. K. Nayar. Polarization-based vision through haze. Applied Optics, 42(3):511-525, 2003. 2, 3

[20] W. A. Shurcliff and S. S. Ballard. Polarized Light, pages 98-103. Van Nostrand, Princeton, N.J., 1964. 2

[21] B. Skerry and H. Hall. Successful Underwater Photography. New York: Amphoto books, 2002. 2, 5

[22] K. Tan and J. P. Oakley. Physics-based approach to color image enhancement in poor visibility conditions. JOSA A, 18(10):2460-2467, 2001. 1

[23] T. Treibitz and Y. Y. Schechner. Instant 3Descatter. In Proc. IEEE CVPR, volume 2, pages 1861-1868, 2006. 3, 8

[24] T. Treibitz and Y. Y. Schechner. Active polarization descattering. IEEE Trans. PAMI, To appear, 2008. 2, 5

[25] T. Treibitz, Y. Y. Schechner, and H. Singh. Flat refractive geometry. In Proc. IEEE CVPR, 2008. 2

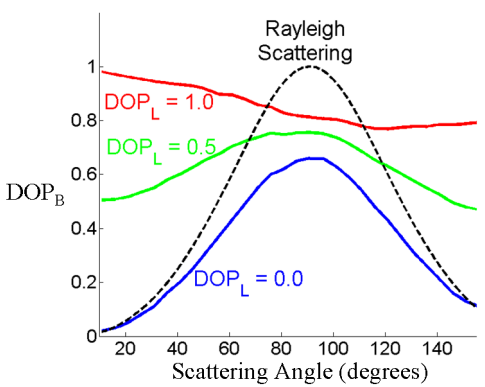

Figure 11. Variation of the DOP of the scattered light, $\mathrm{DOP}_{\mathrm{B}}$, on the scattering angle. For low values of $\mathrm{DOP}_{\mathrm{L}}$, the curve qualitatively resembles that of Rayleigh scattering. For a completely polarized source, the curve is flatter, with an average value of $\approx 0.8$ for backscattering angles $\left(>90^{\circ}\right)$.

[26] H. van de Hulst. Light Scattering by Small Particles. Chapter 5. Wiley, New York, 1957. 8

[27] K. J. Voss and E. S. Fry. Measurement of the mueller matrix for ocean water. Applied Optics, 23:4427-4439, 1984. 7, 8

[28] S. Zhang and S. Negahdaripour. 3D shape recovery of planar and curved surfaces from shading cues in underwater images. IEEE Journal of Oceanic Engineering, 27:100-116, 2002. 1

\section{A. Degree of Polarization of Scattering}

In this appendix, we study the dependence of the DOP of the scattered light, $\mathrm{DOP}_{\mathrm{B}}$, on the scattering angle and the $\mathrm{DOP}$ of the incident light, $\mathrm{DOP}_{\mathrm{L}}$. We consider only the vertical and horizontal polarized components of linearly polarized light. Hence, we consider the first $2 \times 2$ sub-matrix of the full $4 \times 4$ Mueller matrix. Polarization properties of scattered light can be characterized by the Mueller matrix [26]:

$$
\left[\begin{array}{c}
I_{\mathrm{B}} \\
Q_{\mathrm{B}}
\end{array}\right]=\left[\begin{array}{ll}
m_{11} & m_{12} \\
m_{21} & m_{22}
\end{array}\right]\left[\begin{array}{c}
I_{\mathrm{L}} \\
Q_{\mathrm{L}}
\end{array}\right]
$$

where $I_{\mathrm{L}}$ is the sum, and $Q_{\mathrm{L}}$ is the difference of the horizontal and vertically polarized components of the incident light. Similarly, $I_{\mathrm{B}}$ and $Q_{\mathrm{B}}$ are the sum and difference respectively of the scattered light. Note that DOP $=\frac{Q}{I}$. Consequently, based on Eq. (14):

$$
\mathrm{DOP}_{\mathrm{B}}=\frac{m_{21}+m_{22} \mathrm{DOP}_{\mathrm{L}}}{m_{11}+m_{12} \mathrm{DOP}_{\mathrm{L}}} .
$$

Using the above equation and the measured Mueller matrix data for ocean water [27], we plot $\mathrm{DOP}_{\mathrm{B}}$ versus the scattering angle in Figure 11. For comparison, we also plot the behavior for Rayleigh scattering. For low values of $\mathrm{DOP}_{\mathrm{L}}$ (natural light), the curve qualitatively resembles that of Rayleigh scattering. On the other hand, for a completely polarized source (for example, an LCD projector), the curve is flatter, with an average value of 0.8 for backscattering angles. Interestingly, this agrees with the observation made in [23] as well. 